\title{
Signatures of Galaxy Formation in the Intracluster Medium
}

\author{
G. Mark Voit ${ }^{1,2} \&$ Trevor J. Ponman ${ }^{3}$
}

\begin{abstract}
The intergalactic gas in groups and clusters of galaxies bears the indelible stamp of galaxy formation. We present a comparison between observations and simple theoretical models indicating that radiative cooling governs the entropy scale that sets the core radius of the intracluster medium. Entropy measured at the radius $0.1 r_{200}$ scales as $T^{2 / 3}$, in accord with cooling-threshold models for the regulation of core entropy. Cooling of baryons to form galaxies is likely to lead to feedback, and the signature of feedback may appear farther out in the cluster. Entropy measured at the radius $r_{500}$ in all but the most massive clusters exceeds the amount that can be generated by hierarchical accretion. However, feedback that smoothes the density distribution of accreting baryons, perhaps via galactic winds, can boost entropy production at the accretion shock by a factor $\sim 2-4$. An initial comparison of entropy at $r_{500}$ to smooth accretion models shows that smooth accretion is a plausible explanation for this excess entropy and suggests that baryon accretion onto groups was smoother than baryon accretion onto clusters.
\end{abstract}

Subject headings: cosmology: theory — galaxies: clusters: general — galaxies: evolution — intergalactic medium - X-rays: galaxies: clusters

\section{Introduction}

Clusters and groups of galaxies are interesting laboratories for studying the cooling and feedback processes that govern galaxy formation because these non-gravitational processes have clearly altered the structure of intracluster and intragroup media. Simulations show that the density profiles of dark-matter halos created by hierarchical structure formation are nearly self-similar, with a moderate trend for dark matter to be more concentrated toward the centers of lower-mass haloes (e.g., Navarro, Frenk, \& White 1997). The baryonic density profiles of those halos in purely gravitational simulations are also nearly self-similar, leading to an X-ray luminosity-temperature relation that agrees with self-similar scaling: $L_{X} \propto T_{X}^{2}$ in the bremsstrahlung-dominated regime (Muanwong et

\footnotetext{
${ }^{1}$ Department of Physics and Astronomy, Michigan State University, East Lansing, MI 48824, voit@pa.msu.edu

${ }^{2}$ Space Telescope Science Institute

${ }^{3}$ School of Physics and Astronomy, University of Birmingham, Edgbaston, Birmingham B15 2TT, UK, tjp@star.sr.bham.ac.uk
}

al. 2001; Borgani et al. 2001).

Cluster observations showing that $L_{X} \stackrel{\propto}{\sim} T_{X}^{3}$ for $T_{X} \gtrsim 2 \mathrm{keV}$ were the first clear indication that galaxy formation somehow breaks the selfsimilarity of the baryonic component (e.g., Edge \& Stewart 1991; David et al. 1993; Markevitch 1998; Arnaud \& Evrard 1999). This effect shows up even more dramatically in the steeper $L_{X^{-}}$ $T_{X}$ relation for groups (Helsdon \& Ponman 2000) and the surprising faintness of the unresolved $\sim 1 \mathrm{keV}$ background (Pen 1998; Wu, Fabian, \& Nulsen 2001; Bryan \& Voit 2001). Recent observations resolving the temperature structure of clusters have revealed similarity breaking in the mass-temperature relation as well. Self-similar models with $M_{\Delta} \propto T_{X}^{3 / 2}$, where $M_{\Delta}$ is the total mass within a sphere whose mean density is $\Delta$ times the critical density, agree well with purely gravitational simulations (e.g., Evrard, Metzler, \& Navarro 1996; Bryan \& Norman 1998). However, X-ray observations that resolve the temperature structure of clusters now indicate a slightly steeper slope $M_{\Delta} \stackrel{\propto}{\sim} T_{X}^{1.8}$ and a mass normalization $\sim 1.4$ times larger than in simulations without 
galaxy formation (Horner, Mushotzky, \& Scharf 1999; Nevalainen, Markevitch, \& Forman 2000; Finoguenov, Reiprich, \& Böhringer 2001; Sanderson et al. 2003).

Including galaxy formation in the simulations generally steepens the $L_{X}-T_{X}$ relation, but it is not clear whether the dominant mechanism responsible for similarity breaking is cooling or heating. Simulations that preheat the intracluster medium by imposing an entropy floor equivalent to the $100-150 \mathrm{keV} \mathrm{cm}^{2}$ levels observed in the cores of groups by Ponman et al. (1999) produce good agreement with the observed $L_{X}-T_{X}$ relation (Bialek, Evrard, \& Mohr 2001). ${ }^{4}$ Yet, simulations that include radiative cooling without a compensating heat source seem to agree equally well with the data, at the expense of an uncomfortably large condensed baryon fraction ( $\gtrsim 30 \%)$ on group scales (Muanwong et al. 2001; Dave, Katz, \& Weinberg 2002; Tornatore et al. 2003; Kay, Thomas, \& Theuns 2003).

The insensitivity of the $L_{X}-T_{X}$ relation to the details of galaxy formation can be understood in terms of the entropy threshold for cooling within a Hubble time (Voit \& Bryan 2001). Gas at $\sim 1 \mathrm{keV}$ with entropy equivalent to $\lesssim 100 \mathrm{keV} \mathrm{cm}^{2}$ can radiate away its thermal energy in less than a Hubble time. It must either condense or be heated by feedback to a higher entropy level. The cooling threshold therefore imprints a physical entropy scale on a medium that would otherwise be nearly scale-free. A flattening of the entropy distribution below the cooling threshold, not necessarily an absolute entropy floor, is all that is needed to produce the observed $L_{X}-T_{X}$ relation, as long as it forces the cluster's density profile to be flatter than $n(r) \propto r^{-3 / 2}$ where the cooling time is less than a Hubble time. Then, the bulk of the cluster's luminosity comes from regions where the cooling time is similar to a Hubble time, linking the cluster's overall luminosity to the cooling threshold (Voit et al. 2002). Simulations of cluster formation performed with a wide range of feedback efficiencies support this basic picture, as long as that feedback heats the affected gas to $\gtrsim 1 \mathrm{keV}$ (Kay et al. 2003). In order to distinguish the effects of

\footnotetext{
${ }^{4}$ Entropy in this paper is quantified in terms of the X-ray observable $K=T n_{e}^{-2 / 3}$, where $n_{e}$ is the electron density. The standard specific entropy scales with the logarithm of this quantity.
}

cooling from those of heating, more information is needed, and some of that information can be found in observations that resolve the entropy profiles of clusters and groups (e.g., Ponman et al. 2003), which depend in detail on the interplay between cooling, heating, and hierarchical accretion (Voit et al. 2003).

The purpose of this Letter is to highlight two trends found in those spatially resolved entropy observations and to present an initial comparison with theoretical predictions by way of simple analytical models. Section 2 focuses on the core entropy measured at $0.1 r_{200}$, where $r_{200}$ is the radius at which $\Delta=200$. Early measurements of core entropy suggested that a global entropy floor $\sim 135 \mathrm{keV} \mathrm{cm}^{2}$ applied to all groups and clusters (Ponman et al. 1999; Lloyd-Davies, Ponman, \& Cannon 2000), but updated measurements indicate that entropy at $0.1 r_{200}$ scales as $T^{2 / 3}$ (Ponman et al. 2003), as expected if the cooling threshold indeed determines core entropy. Section 3 focuses on entropy measured at $r_{500}$, where $\Delta=500$. Somewhat unexpectedly, entropy at these much larger radii also exceeds the predictions of self-similar models, sometimes by as much as $\sim 10^{3} \mathrm{keV} \mathrm{cm}^{2}$ (Finoguenov et al. 2002; Ponman et al. 2003). Comparing these measurements to analytical models for smooth accretion suggests that smoothing of the intergalactic medium before it accretes onto a cluster or group is a promising mechanism for producing this entropy excess (Voit et al. 2003; Ponman et al. 2003). In $\S 4$ we discuss the implications of these results and offer a few hypotheses to test with numerical simulations.

\section{Core Entropy and Cooling}

The entropy of intracluster and intragroup media is generated primarily by shocks owing to mergers and accretion. Simulations show that these gravitationally driven processes lead to an entropy profile $K(r) \stackrel{\propto}{\sim} r^{1.1}$ (Borgani et al. 2001), a scaling that reflects the history of mass accretion onto the cluster (Tozzi \& Norman 2001; Voit et al. 2003). Alterations of that profile owing to nongravitational heating and radiative cooling should be most obvious at small radii, where the gravitationally generated entropy is smallest. Models involving strong global preheating typically predict that cluster cores should be nearly isentropic, 


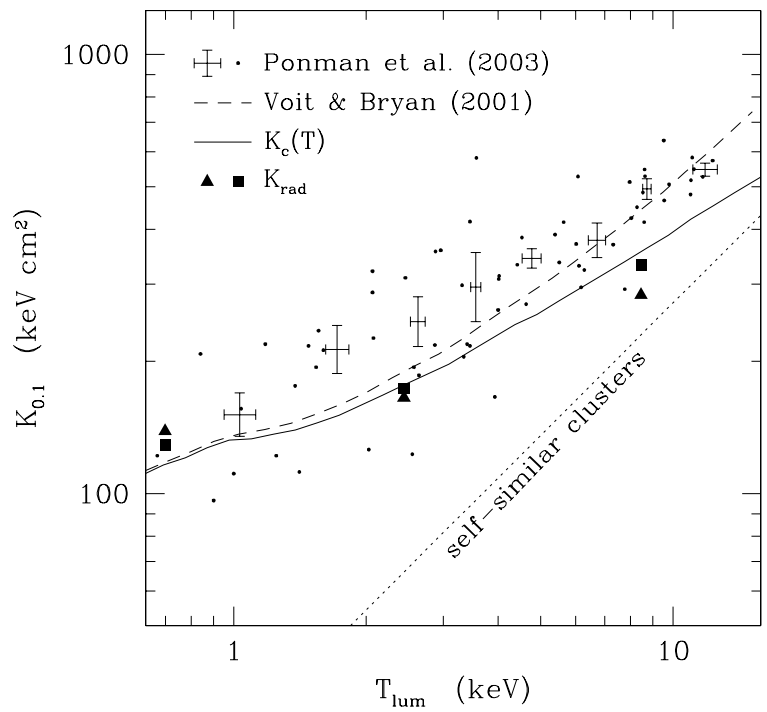

Fig. 1.- Relationship between core entropy and the cooling threshold. Each point with error bars shows the mean core entropy $K_{0.1}$, measured at $0.1 r_{200}$, for eight clusters within a given bin of luminosity-weighted temperature $T_{\text {lum }}$, and small circles show measurements for individual clusters (Ponman et al. 2003). The dotted line shows a selfsimilar relation calibrated using the median value of $K_{0.1}$ measured in simulation L50+ of Bryan \& Voit (2001) which does not include cooling or feedback. The solid line shows the cooling threshold $K_{c}(T)$, defined to be the entropy at which the cooling time equals $14 \mathrm{Gyr}$, assuming the cooling function of Sutherland \& Dopita (1993) for 0.3 solar metallicity. The dashed line shows the entropy at $0.1 r_{200}$ in the model of Voit \& Bryan (2001) when this cooling function is used. Solid points without error bars show the total radiative entropy loss $K_{\text {rad }}$ computed for individual halos with the accretion histories of Tozzi \& Norman (2001, squares) and Voit et al. (2003, triangles). These simple treatments of cooling reproduce the slope but slightly underestimate the normalization of the observed $K_{0.1}\left(T_{\text {lum }}\right)$ relation, which shows no sign of an absolute entropy floor.

at the level of the global entropy floor (Balogh, Babul, \& Patton 1999; Tozzi \& Norman 2001; Babul et al. 2002). In models that rely more on cooling to break self-similarity, the details of the core entropy profile depend on the interplay between cooling, feedback, and merging, and perhaps on conduction as well (Voit et al. 2002). Regardless of these details, the entropy scale of similarity breaking set by cooling should correspond to the cooling threshold $K_{c}(T) \propto T^{1 / 3} \Lambda^{2 / 3}$, which leads to $K_{c} \propto T^{2 / 3}$ when bremsstrahlung dominates the cooling function $\Lambda$.

Recent observational evidence favors cooling- threshold models. Figure 1 shows measurements of core entropy $K_{0.1}$ drawn from a compilation of cluster observations. Ponman et al. (2003) collected data from 64 clusters and groups and presented the mean entropy levels within temperature bins containing eight clusters each. Binning the data averages out the cluster-to-cluster variations, which can range up to a factor of three at a given temperature (e.g., Mushotzky et al. 2003). These measurements sit well above the dotted line showing a self-similar relation calibrated to match the purely gravitational simulations of Bryan \& Voit (2001). However, the measurements do not level off at a well-defined entropy floor. Instead, they trace a power-law relation $K_{0.1} \propto T^{0.65}$, nearly identical to the scaling of the cooling threshold (Ponman et al. 2003).

Entropy profiles of individual clusters and groups likewise show no evidence for an absolute entropy floor. The cores of groups observed with $X M M$-Newton are not isentropic but instead have entropy profiles similar to those of clusters (Mushotzky et al. 2003; Pratt \& Arnaud 2003). In fact, scaling those profiles according to $K \propto T^{2 / 3}$ produces a much closer match to massive-cluster profiles than the expected selfsimilar scaling $K \propto T$ (Pratt \& Arnaud 2003; Ponman et al. 2003). Furthermore, the core entropy levels required to explain the $L_{X}-T_{X}$ relation with isentropic models are far higher than those observed (Voit et al. 2003).

Simplistic models involving the cooling threshold come close to matching the $K_{0.1}-T_{\text {lum }}$ relation, but the match is not exact. The solid line in Figure 1 shows the cooling threshold $K_{c}\left(T_{\text {lum }}\right)$, defined to be the entropy level at which the cooling time equals $14 \mathrm{Gyr}$, according to the cooling function for 0.3 solar metallicity from Sutherland \& Dopita (1993). This threshold lies $\sim 25 \%$ below the binned data points with a very similar slope. The dashed line shows $K_{0.1}$ computed from $K_{c}$ using the prescription of Voit \& Bryan (2001; see also Wu \& Xue 2002a,b). In these models the self-similar entropy distribution of unmodified intracluster gas is simply truncated below $K_{c}$, and the gas is allowed to relax back into hydrostatic equilibrium. These truncated models agree better with the hotter clusters but not do not provide much enhancement over $K_{c}$ near $\sim 1 \mathrm{keV}$ because this simplistic prescription leads to nearly 
isentropic cores in the low-mass clusters. Treating cooling more realistically produces an entropy gradient within $0.1 r_{200}$ that declines to zero entropy at the origin, in the absence of feedback and conduction (Voit et al. 2002).

A more detailed computation of $K_{c}$ accounting for time dependence of the gas temperature does not change this basic result. One can compute the total entropy loss $K_{\text {rad }}$ owing to radiative cooling assuming that the gas temperature at a given time equals the temperature of the most massive progenitor halo at that time (see Voit et al. 2003). The squares and triangles in Figure 1 show that $K_{\text {rad }}$ differs little from $K_{c}$ for a typical merger history (see also Oh \& Benson 2003).

Taken as whole, Figure 1 strongly suggests that radiative cooling regulates the entropy scale that defines the cores of clusters. However, the large dispersion of the unbinned data points indicates that cooling acts in concert with other processes. Much of that dispersion may arise from the stochastic nature of mergers, which produce significant entropy differences in clusters of similar temperature (see Figure 5 of Voit et al. 2003). Differing feedback histories may also be responsible for some of this dispersion.

\section{Outer Entropy and Smoothing}

Elevated entropy levels in cluster cores were once thought to be evidence for non-gravitational energy input into the intracluster medium, but the close link between core entropy and the cooling threshold now renders that interpretation of core entropy somewhat questionable. Nevertheless, feedback from galaxy formation still seems necessary to limit the fraction of condensed baryons in clusters. In models of hierarchical structure formation, a large percentage of the plasma that will become the intracluster medium has a cooling time less than a Hubble time at $z \sim 2-3$ (e.g., Voit et al. 2002). Because only $\sim 5-10 \%$ of baryons are observed to be in stars (e.g., Balogh et al. 2001), feedback from supernovae and active galactic nuclei presumably intervenes to prevent most of this intergalactic gas from condensing. Here we present evidence suggesting that this early episode of cooling and feedback may couple with subsequent merging and accretion to produce an observable entropy signature in the outskirts of clusters owing to the smoothing effect of galactic feedback on the intergalactic medium.

Hierarchical merging in the absence of cooling and feedback tends to produce a nearly self-similar entropy distribution (Voit et al. 2003). The characteristic entropy associated with the baryons in a halo of mass $M_{200}$ is $K_{200} \equiv T_{200} \bar{n}_{e}^{-2 / 3}$, where $T_{200}=G M_{200} \mu m_{p} / 2 r_{200}$ is the characteristic temperature of the halo and $\bar{n}_{e} \approx 1.6 \times 10^{-4} \mathrm{~cm}^{-3}$ is $200 \Omega_{M}^{-1}$ times the mean electron density of the universe. According to the simulations of Bryan $\&$ Voit (2001), the maximum value of intracluster entropy is typically $\approx 0.8 K_{200}$ without galaxy formation, and the median entropy value at the scale radius $r_{500}$ is $\approx 0.4 K_{200}$. These scalings do not appear to depend significantly on halo mass.

In contrast, observations imply a considerably higher entropy value at $r_{500}$ with a clear dependence on halo mass (Finoguenov et al. 2002; Ponman et al. 2003). Figure 2 shows mean values of $K\left(r_{500}\right)$ inferred by Ponman et al. (2003) from the same data set as in Figure 1. Notice that all of the binned data points lie above the dotted line showing the value of $K\left(r_{500}\right)$ predicted by selfsimilar models calibrated with the simulations of Bryan \& Voit (2001), indicating that galaxy formation affects the entire entropy profile of a cluster, not just the core. The observed entropy boost is quite large. A solid line showing the value of $K_{200}$ inferred from $T_{\text {lum }}$ and the observed $M_{200^{-}}$ $T_{\text {lum }}$ relation of Sanderson et al. (2003) indicates the maximum entropy expected from pure hierarchical accretion. All the data points below $\sim 5 \mathrm{keV}$ lie above this line by hundreds of $\mathrm{keV} \mathrm{cm}^{2}$, representing an entropy excess that is very difficult to produce with non-gravitational energy injection alone (Ponman et al. 2003).

Voit et al. (2003) and Ponman et al. (2003) independently suggested that this excess entropy arises because feedback has smoothed the baryonic matter that accretes onto a cluster, thereby reducing the characteristic density of gas that passes through the accretion shock. Smoothing boosts intracluster entropy because the entropy of strongly shocked gas is $\propto v_{s}^{2} \rho_{1}^{-2 / 3}$, where $v_{s}$ is the shock velocity and $\rho_{1}$ is the preshock baryon density. The scale of $v_{s}$ is determined by dark-matter dynamics that are virtually unaffected by cooling and feedback. Thus, feedback raises the mass-weighted av- 


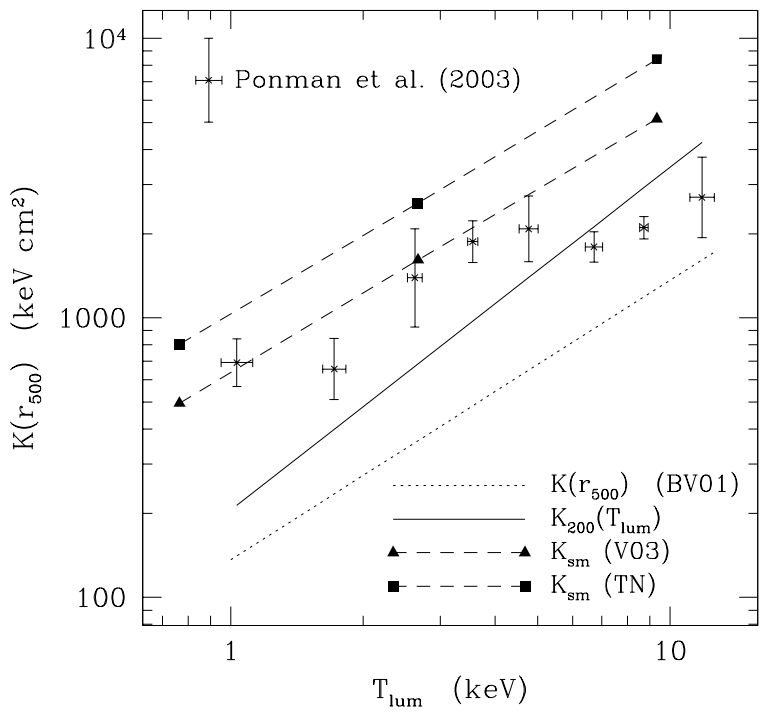

Fig. 2.- Entropy at $r_{500}$ as a function of luminosityweighted cluster temperature. Each point with error bars shows the mean value of $K\left(r_{500}\right)$ implied by the density and temperature profiles of eight clusters within that temperature bin (Ponman et al. 2003). The dotted line shows the predicted entropy at $r_{500}$ for self-similar clusters calibrated with simulation L50+ of Bryan \& Voit (2001). The solid line shows the entropy scale $K_{200}$ computed from $T_{\text {lum }}$ using the $M_{200}-T_{\text {lum }}$ relation of Sanderson et al. (2003), which is the maximum amount of intracluster entropy expected from hierarchical accrection in the absence of nongravitational processes (see Voit et al. 2003). Dashed lines connect the maximum entropy values produced by smooth accretion onto individual halos, according the accretion histories of Voit et al. (2003, triangles) and Tozzi \& Norman (2001, squares). The data suggest a transition from hierarchical accretion in the most massive clusters to smooth accretion on group scales.

erage value of $v_{s}^{2} \rho_{1}^{-2 / 3}$ because it reduces the massweighted average value of $\rho_{1}$ (Voit et al. 2003). Notice that a modest amount of preshock entropy can be strongly amplified at the accretion shock of a deep potential well if that preshock entropy is sufficient to drive a large proportion of baryons out of the shallower potential wells of the accreting dark matter. Ponman et al. (2003) envisioned this smoothing as a thickening of the filaments through which matter accretes. Voit et al. (2003) modeled this entropy increase by developing analytical models for entropy production during smooth accretion.

The models of Voit et al. (2003) show that smooth, spherically symmetric accretion produces an entropy profile whose shape is similar to that found in simulations without galaxy formation but whose normalization is $\sim 2$ times higher in massive clusters and up to $\sim 4$ times higher in groups. The magnitude of this entropy excess depends on the mass-accretion rate - objects that accrete most of their mass late in time have a higher baryon density entering the accretion shock and therefore less of an entropy excess. The dashed lines in Figure 2 indicate the maximum amount of entropy produced by smooth accretion, assuming the accretion histories of Tozzi \& Norman (2001; squares) and Voit et al. (2003; triangles) for halo masses of $10^{13} h^{-1} M_{\odot}, 10^{14} h^{-1} M_{\odot}$, and $10^{15} h^{-1} M_{\odot}$.

This initial comparison between analytical models for smooth accretion and observed entropy levels at $r_{500}$ suggest that smoothed accretion is a plausible explanation for these entropy excesses because the data points in Figure 2 approach but do not exceed the dashed lines. Furthermore, the trend of the data points with $T_{\text {lum }}$ suggests that accretion of baryons onto groups is smoother than accretion of baryons onto clusters. This apparent transition from nearly smooth accretion at $\lesssim 1 \mathrm{keV}$ to purely hierarchical accretion at $\gtrsim 10 \mathrm{keV}$ may also explain why the entropy profiles of groups are similar in shape to those of clusters but have shifted normalizations (e.g., Pratt \& Arnaud 2003). Curiously, scaling the profiles by the same $T^{2 / 3}$ factor found in the cores brings them into agreement, suggesting that the cooling threshold plays a role in setting the smoothing scale. However, it is not immediately clear how cooling and feedback would couple with accretion to produce this scaling.

\section{Discussion}

The entropy of baryons in both the cores and outskirts of clusters disagrees with simulations that do not include galaxy formation. Thus, the entropy profiles of these objects contain valuable information about the impact of galaxy formation on the intergalactic medium and the role of feedback in regulating global star formation. The scaling of core entropy $\left(K_{0.1} \propto T_{\text {lum }}^{2 / 3}\right)$ suggests that it is governed by radiative cooling (Ponman et al. 2003). However, feedback is likely to limit the fraction of baryons that eventually cool and form stars.

If feedback is strong enough to eject a sub- 
stantial percentage of baryons from low-mass halos, then this smoothing of the baryon distribution will affect entropy production during subsequent accretion and mergers. Comparing smoothaccretion models with the available data (Figure 2) suggests that a large percentage of the matter accreting onto groups and low-mass clusters must be smoothed in order to explain the observed entropy excess at $r_{500}$. If this explanation is correct, then smoothing may also have an observable impact on the X-ray substructure in and around low-mass clusters and the associated filaments. Measuring how feedback affects the level of X-ray substructure in numerically simulated groups and clusters would help observers to quantify the level of intergalactic smoothing.

Only in the highest-mass clusters do entropy profiles approach the self-similar form predicted by simulations without cooling and feedback. However, these clusters presumably formed through mergers of lower-mass objects in which cooling and feedback had already broken self-similarity. We speculate that hierarchical merging restores similarity because the self-similar entropy profile behaves as a dynamical attractor: As structure grows, the deepening potential wells are increasingly able to overcome baryon smoothing produced by early feedback. Accretion onto massive clusters is therefore lumpier than accretion onto groups, and the resulting entropy profile approaches self-similarity when the accreting lumps of dark matter are able to retain the majority of their associated baryons.

Many questions about the link between galaxy formation and the intracluster medium remain unanswered. Among the most crucial is the amount of energy injection implied by the entropy profiles of clusters. Numerical simulations that track entropy production in low-mass halos would be very helpful because smoothing allows a small amount of entropy injection to be amplified with each merger event during the formation of a group or cluster. High resolution simulations will therefore be needed to assess how early feedback propagates through the entire merger hierarchy and to characterize how the hottest clusters eventually approach self-similarity.

The authors thank Greg Bryan for sharing his insights into simulated clusters and Alistair
Sanderson for his contributions to the analysis of the observational data. GMV received partial support from NASA through grant NAG5-3257.

\section{REFERENCES}

Arnaud, M., \& Evrard, A. E. 1999, MNRAS, 305, 631

Babul, A., Balogh, M. L., Lewis, G. F., \& Poole, G. B. 2002, MNRAS, 330, 329

Balogh, M. L., Babul, A., \& Patton, D. R. 1999, MNRAS, 307, 463

Balogh, M. L., Pearce, F. R., Bower, R. G., \& Kay, S. T. 2001, MNRAS, 326, 1228

Bialek, J. J., Evrard, A. E., \& Mohr, J. J. 2001, ApJ, 555, 597

Borgani, S., Governato, F., Wadsley, J., Menci, N., Tozzi, P., Lake, G., Quinn, T., \& Stadel, J. 2001, ApJ, 559, L71

Bower, R. G., Benson, A. J., Lacey, C. G., Baugh, C. M., Cole, S., \& Frenk, C. S. 2001, MNRAS, 325,497

Bryan, G. L., \& Norman, M. L. 1998, ApJ, 495, 80

Bryan, G. L., \& Voit, G. M. 2001, ApJ, 556, 590

Davé, R., Katz, N., \& Weinberg, D. H. 2002, ApJ579, 23

David, L. P. et al. 1993, ApJ, 412, 479

Edge, A. C., \& Stewart, G. C. 1991, MNRAS, 252, 414

Evrard, A. E., \& Henry, J. P. 1991, ApJ, 383, 95

Evrard, A. E., Metzler, C., \& Navarro, J. F. 1996, ApJ, 469, 494

Finoguenov, A., Reiprich, T. H., \& Böhringer, H. 2001, A\&A, 368, 749

Finoguenov, A., Jones, C., Böhringer, H., Ponman, T. J. 2002, ApJ, 578, 74

Helsdon, S. F., \& Ponman, T. J. 2000, MNRAS, 315,356 
Horner, D. J., Mushotzky, R. F., \& Scharf, C. A. 1999, ApJ, 520, 78

Kay, S. T., Thomas, P. A., \& Theuns, T. 2003, MNRAS, in press (astro-ph/0210560)

Lloyd-Davies, E. J., Ponman, T. J., \& Cannon, D. B. 2000, MNRAS, 315, 689

Markevitch, M. 1998, ApJ, 504, 27

Mushotzky, R., Figueroa-Feliciano, E., Loewenstein, M., Snowden, S. L. 2003, astro$\mathrm{ph} / 0302267$

Muanwong, O., Thomas, P. A., Kay, S. T., Pearce, F. R., \& Couchman, H. M. P. 2001, ApJ, 552, $27 \mathrm{~L}$

Navarro, J. F., Frenk, C. S., \& White, S. D. M. 1997, ApJ, 490, 493

Nevalainen, J., Markevitch, M., \& Forman, W. 2000, ApJ, 532, 694

Oh, S. P., \& Benson, A. 2002, astro-ph/0212357

Ponman, T. J., Cannon, D. B., \& Navarro, J. F. 1999, Nature, 397, 135

Ponman, T. J., Sanderson, A. J. R., \& Finguenov, A. 2003, MNRAS, in press (astro-ph/0304048)

Pratt, G., \& Arnaud, M. 2003, astro-ph/0304017

Sanderson, A. J. R., Ponman, T. J., Finoguenov, A., Lloyd-Davies, E. J., \& Markevitch, M. 2003, MNRAS, in press (astro-ph/0301049)

Sutherland, R. S., \& Dopita, M. A. 1993, ApJS, 88,253

Tornatore, L., Borgani, S., Springel, V., Matteucci, F., Menci, N., \& Murante, G. 2003, MNRAS, sumbitted (astro-ph/0302575)

Tozzi, P., \& Norman, C. 2001, ApJ, 546, 63

Voit, G. M., Balogh, M. L., Bower, R. G., Lacey, C. G., \& Bryan, G. L., 2003, ApJ, in press (astro-ph/0304447)

Voit, G. M., \& Bryan, G. L. 2001, Nature, 414, 425

Voit, G. M., Bryan, G. L., Balogh, M. L., \& Bower, R. G. 2002, ApJ, 576, 601
Wu, K. K. S., Fabian, A. C., \& Nulsen, P. E. J. 2001, MNRAS, 324, 95

Wu, X.-P., \& Xue, Y.-J. 2002a, ApJ, 569, 112

Wu, X.-P., \& Xue, Y.-J. 2002b, ApJ, 572, 19

This 2-column preprint was prepared with the AAS IATEX macros v5.0. 